\title{
Patients with Borderline Personality Disorder Not Participating in an RCT: Are They Different?
}

\author{
Michael Rentrop ${ }^{a} \quad$ Philipp Martius $^{c}$ Josef Bäuml ${ }^{a}$ Peter Buchheim ${ }^{a}$ \\ Stephan Döring ${ }^{d-f}$ Susanne Hörz ${ }^{a, b}$ \\ ${ }^{a}$ Department of Psychiatry and Psychotherapy, Technical University of Munich, and ${ }^{b}$ Department of Psychology, \\ Ludwig Maximilians University Munich, Munich, ' ${ }^{C}$ linik Höhenried, Bernried, and ${ }^{\mathrm{d}}$ Psychosomatics in Dentistry, \\ Department of Prosthodontics and Material Sciences, and ${ }^{\mathrm{E}}$ Department of Psychosomatics an Psychotherapy, \\ University of Münster, Münster, Germany; ${ }^{f}$ Department of Medical Psychology and Psychotherapy, \\ Medical University, Innsbruck, Austria
}

\section{Key Words}

Randomized controlled trial · Borderline personality

disorder $\cdot$ External validity

\begin{abstract}
Background: Despite the notion that randomized controlled trials are regarded as the gold standard in psychotherapy research, questions about their generalizability have been raised. This paper focuses on the differences between participants and eligible nonparticipants of a randomized controlled trial for patients with borderline personality disorder (BPD). Sampling and Methods: One hundred forty-two patients were screened, and 122 were found eligible for study participation. Out of these, 64 patients (52.5\%) gave informed consent and were included in the study. $\boldsymbol{R} \boldsymbol{e}$ sults: The 58 eligible nonparticipants showed a lower level of functioning (global assessment of functioning score), had a history of more outpatient treatment attempts and were living alone more often. Regarding acute symptoms and severity of BPD as indexed by suicide attempts, inpatient treatments, substance abuse and history of trauma, no differences between the groups could be detected. Moreover, participants showed significantly more eating disorders,
\end{abstract}

whereas nonparticipants presented more affective and anxiety disorders. Conclusions: The results indicate that lower psychosocial functioning and comorbid affective and anxiety disorders decrease BPD patients' willingness to participate in an RCT.

Copyright $\odot 2010$ S. Karger AG, Basel

\section{Introduction}

In the last 15 years major randomized controlled trials (RCTs) on treatments for borderline personality disorder (BPD) have been published [e.g. 1-6]. These studies rarely gave detailed information on their recruitment procedures. As a consequence, there is little knowledge about patients who were eligible for the major RCTs but not willing to take part. Studying these patients is of special interest since it helps close the gap between RCT populations and clinical reality [7].

This study was funded by the Austrian National Bank, Jubiläumsfonds No. 10636.

\section{KARGER}

Fax +41613061234 E-Mail karger@karger.ch www.karger.com
Dr. Michael Rentrop

Department of Psychiatry and Psychotherapy

Klinikum rechts der Isar, Ismaningerstrasse 22, DE-81675 Munich (Germany)

Tel. +49 894140 4265, Fax +49 8941404888

E-Mail michael.rentrop@lrz.tu-muenchen.de 
This paper investigates the differences between participants and eligible nonparticipants of an RCT for the treatment of BPD and the factors that influence a patient's willingness to participate in the RCT. Based on our clinical experience and the very scarce pool of literature on this topic [e.g. 8], we tested the hypothesis that the group of patients who denied participation in the study showed significantly more pathology than the participants.

\section{Method}

\section{Background}

This study was performed as part of a German/Austrian multicenter RCT [9] comparing the effectiveness of transference-focused psychotherapy [10] to treatment delivered by experienced community psychotherapists for individuals with BPD. The inclusion and exclusion criteria are summarized in table 1.

\section{Procedure}

The study was approved by the local ethics committee. The study recruitment took place at the Outpatient Department of a German University Psychiatric Hospital from October 2004 to July 2006. Most patients came on their own initiative or on referral from psychiatric treatment settings (e.g. crisis intervention units, psychiatric hospitals, private practices). During the initial psychiatric interview, the patients were informed about the study procedures and details as well as alternatives to study participation. A notion of preference for 1 of the 2 treatment approaches was strictly avoided. Additionally, the initial psychiatric interview was designed as a clinical screening instrument for study inclusion and exclusion criteria. The interviews lasted about 60 min, and all study interviews were performed by the same psychiatrist (M.R.). Axis I disorders were clinically diagnosed according to DSM-IV criteria and the global assessment of functioning score (GAF; [11]) was recorded. Regarding axis II disorders, the patients received diagnoses based on clinical judgment, focusing on BPD and antisocial personality disorder exclusively. The patients were additionally asked about psychiatric disorders of parents and relatives. Their history of traumatic events was explored in a nonstandardized format. For this, the interviewer typically explored the nature of the trauma and the age at which it occurred. The nature of the trauma was coded as (a) sexual, (b) physical and (c) emotional, and for quantitative analyses, multiple traumatizations were assigned to the category corresponding to the severest trauma $(\mathrm{a}>\mathrm{b}>\mathrm{c})$. Demographic information was obtained from the routine documentation in the patient charts.

\section{Data Analysis}

The statistical analyses were carried out with SPSS for Windows (version 14.0). Group differences on continuous data were examined by 2 -sided $t$ tests. All variables were tested with regard to normal distribution (Kolmogorov-Smirnov test; $\mathrm{p}<0.05$ ). For nonnormally distributed variables, the results were confirmed with Kruskal-Wallis $\mathrm{H}$ tests. Group differences on categorical data were examined with $\chi^{2}$ tests.
Table 1. Inclusion and exclusion criteria

Inclusion criteria

Female gender

Age between 18 and 45 years

BPD according to DSM IV

Sufficient knowledge of the German language

Exclusion criteria

Lifetime diagnosis of schizophrenia

Present major affective disorder

Present substance dependency

Obvious mental retardation

Antisocial personality disorder

\section{Results}

One hundred forty-two patients were screened. Out of these, study participation was offered to 122 female patients with a clinical diagnosis of BPD. Eleven patients showed exclusion criteria: 2 were male, 8 were diagnosed as having present alcohol or substance addiction, and 1 patient suffered from schizophrenia. Moreover, 9 patients did not fulfill the diagnostic criteria of BPD according to DSM-IV.

Sixty-four patients (52.5\%) agreed to participate, and 58 (47.5\%) rejected the offer to take part in the study. After completing the assessment procedure, none of the 64 participants had to be rejected due to clinically undetected exclusion criteria. Among the 58 nonparticipants, 20 patients $(34.5 \%)$ were interested in a specific treatment, e.g. DBT, which was not offered in the study protocol. Six patients $(10.3 \%)$ did not want to give up the therapist treating them before study enrollment. Three individuals (5.2\%) did not agree to be randomized, 5 patients (8.6\%) were searching for inpatient treatment due to a crisis situation, and 24 individuals (41.4\%) stopped contacting the research team for unknown reasons.

Group differences with regard to symptom severity, comorbidity, trauma history and family history are displayed in table 2 .

Nonparticipants yielded a significantly lower GAF score in the assessment. Only 1 patient with a GAF score $<40$ was willing to participate, whereas 16 patients in the group of nonparticipants were assigned a GAF score $<40$. In terms of demographic variables, only the patients' living situation was different; nonparticipants were more likely to live alone $(\mathrm{p}<0.01)$. With respect to education and employment situation, there was a trend below the level of statistical significance: participants showed a 
Table 2. Comparison between nonparticipants and participants

\begin{tabular}{|c|c|c|c|c|}
\hline & $\begin{array}{l}\text { Nonparticipants } \\
(\mathrm{n}=58)\end{array}$ & $\begin{array}{l}\text { Participants } \\
(\mathrm{n}=64)\end{array}$ & $\chi^{2}$ & $\mathrm{p}$ value \\
\hline GAF & $42.4 \pm 7.2$ & $52.4 \pm 6.8$ & & $<0.001$ \\
\hline Outpatient psychotherapies & $1.9 \pm 2.3$ & $1.2 \pm 1.2$ & & $<0.05$ \\
\hline Psychiatric inpatient treatments & $3.2 \pm 3.7$ & $2.4 \pm 2.5$ & & n.s. \\
\hline Suicide attempts & $1.4 \pm 3.3$ & $1.4 \pm 2.1$ & & n.s. \\
\hline Age, years & $30.4 \pm 9.4$ & $28.9 \pm 7.1$ & & n.s. \\
\hline Age at first diagnosis of psychiatric symptoms, years & $19.6 \pm 7.2$ & $19.2 \pm 7.2$ & & n.s. \\
\hline Age at diagnosis of BPD & $25.4 \pm 8.2$ & $25.7 \pm 6.9$ & & n.s. \\
\hline Present comorbidity & $26(43)$ & $26(41)$ & & n.s. \\
\hline Major depressive disorder & $6(10)$ & $1(2)$ & $11.93($ d.f. $=4)$ & $<0.5$ \\
\hline Eating disorders & $13(22)$ & $23(36)$ & $10.20($ d.f. $=7)$ & $<0.5$ \\
\hline Present and lifetime diagnosis of substance abuse & $31(53)$ & $35(55)$ & & n.s. \\
\hline History of trauma (sexual, physical, emotional) & $36(62)$ & $40(63)$ & $2.24($ d.f. $=3)$ & n.s. \\
\hline \multicolumn{5}{|l|}{ Psychiatric disorders in relatives } \\
\hline Parents & $24(41)$ & $31(48)$ & $3.36($ d.f. $=5)$ & n.s. \\
\hline Grandparent, uncle/aunt, sibling & $12(21)$ & $20(31)$ & 4.55 (d.f. $=5$ ) & n.s. \\
\hline
\end{tabular}

Results are expressed as means \pm SD or numbers with percentages in parentheses.

slightly higher level of education (higher level of school degree 32.8 vs. $\left.22.4 \%, \chi^{2}=7.31, p=0.19\right)$ and were working full time more frequently (work full time 31.2 vs. $12.1 \%, \chi^{2}=11.92, \mathrm{p}=0.06$ ).

\section{Discussion}

This paper explores the differences between study participants and nonparticipants in an RCT for BPD patients. Two major findings emerge from this study. The first one is that borderline patients who did not participate in the RCT did not differ from participants in acute symptoms and severity of BPD nor in factors often regarded relevant to the etiology of BPD (e.g. suicide attempts, substance abuse, history of trauma, parents' and relatives' psychiatric disorders, as well as educational and occupational situation). The second finding is that nonparticipants were different from patients who chose to participate in variables of general functioning and comorbidity. Eligible nonparticipants showed worse psychosocial functioning and a higher number of previous outpatient psychotherapies, and tended to live alone more frequently than participants. They also showed different comorbid axis I disorders, with nonparticipants presenting pathology in the areas of affective and anxiety disorders, whereas participants more frequently suffered from eating disorders. We conclude that especially patients with a GAF score $<40$ may be at the limit of outpatient treatability and can be considered as a group of individuals more often in need of hospitalization, perhaps with subsequent outpatient treatment.

The group of patients who finally participate in an RCT is determined by several steps in the patient flow. First, inclusion and exclusion criteria filter out a group of patients and then, the patients' willingness to participate yields the final sample. The external validity of a study can be limited by rigid inclusion and exclusion criteria, which in turn makes inclusion unlikely for a vast majority of the ordinary clinical population [12-14]. With a proportion of $86 \%$ (122 of 142) of all screened patients found eligible for study participation, our enrolment protocol seems to be acceptably close to 'real-life' conditions. This is remarkably higher than the ratio reported by Clarkin et al. [4] in a study with a similar design. In this study 207 patients were screened and only 109 were offered study participation, resulting in a ratio of $52.7 \%$ [15].

Still there are some serious limitations to the approach presented in this paper. First, the diagnosis of the group of nonparticipants is based on a clinical interview only. This makes the assumed diagnosis of a BPD likely but not confirmed by standardized instruments. While planning this study we found it difficult to implement a more complicated design due to the fact that the vast majority of nonparticipants was not willing to undergo any scientific protocol, making it nearly impossible to collect as- 
sured data on this special subgroup of patients. Second, the part of patients, who - after the first interview - never contacted the research team again (24 individuals) is rather high, which might reflect that study participation was 'not attractive to patients'. It may well be due to the fact that the German health care system provides quite some comfort to patients seeking psychotherapy. Besides taking over the therapist fees, health insurance concedes the chance to meet several therapists in up to 5 sessions on a probationary basis. To our knowledge, many of the potential study participants were in this process of find- ing the therapist who really 'fits' and study participation was just one possibility of finding treatment among many. There may also be other aspects in terms of requirements to participate in our study that might have deterred patients from participation.

Further research should study eligible nonparticipants as well as dropouts in detail in order to help identify socalled endophenotypes of BPD [16] as a basis for the development of more syndrome-tailored treatment approaches.

\section{References}

1 Bateman A, Fonagy P: Effectiveness of partial hospitalization in the treatment of borderline personality disorder: a randomized controlled trial. Am J Psychiatry 1999;156: 1563-1569.

2 Bateman A, Fonagy P: Randomized controlled trial of outpatient mentalizationbased treatment versus structured clinical management for borderline personality disorder. Am J Psychiatry 2009;166:1355-1364.

3 Blum N, John DS, Pfohl B, Stuart S, McCormick B, Allen J, Arndt S, Black DW: Systems Training for Emotional Predictability and Problem Solving (STEPPS) for outpatients with borderline personality disorder: a randomized controlled trial and 1-year followup. Am J Psychiatry 2008;165:468-478.

4 Clarkin JF, Levy KN, Lenzenweger MF, Kernberg OF: Evaluating three treatments for borderline personality disorder: a multiwave study. Am J Psychiatry 2007;164:922928.

5 Giesen-Bloo J, van Dyck R, Spinhoven P, van Tilberg W, Dirksen C, van Asselt T, et al: Outpatient psychotherapy for borderline personality disorder: randomized trial of schema-focused therapy vs. transference-focused psychotherapy. Arch Gen Psychiatry 2006;63:649-658.
6 Linehan MM, Comtois KA, Murray AM, Brown MZ, Gallop RJ, Heard HL, et al: Twoyear randomized controlled trial and followup of dialectical behavior therapy vs. therapy by experts for suicidal behavior and borderline personality disorder. Arch Gen Psychiatry 2006;63:757-766.

7 Gross CP, Mallory R, Heiat A, Krumholz HM: Reporting the recruitment process in clinical trials: who are these patients and how did they get there? Ann Intern Med 2002;137:10-16.

8 Van Heuvelen MJ, Hochstenbach JB, Brouwer WH, de Greef MH, Zijilstra GA, van Jaarsveld E, et al: Differences between participants and non-participants in an RCT on physical activity and psychological interventions for older persons. Aging Clin Exp Res 2005; 17:236-245.

9 Doering S, Hörz S, Rentrop M, Fischer-Kern M, Schuster P, Benecke C, et al: Transference-focused psychotherapy vs. treatment by community psychotherapists for borderline personality disorder: randomised controlled trial. Br J Psychiatry 2010;196:389-395.

10 Clarkin JF, Yeomans FE, Kernberg OF: Psychotherapy for Borderline Personality: Focusing on Object Relations. Washington, American Psychiatric Publishing Inc, 2006.
11 Endicott J, Spitzer RL, Fleiss JL, Cohen J: The global assessment scale: a procedure for measuring overall severity of psychiatric disturbance. Arch Gen Psychiatry 1976;3:766-771.

12 Begg C, Cho M, Eastwood S, Horton R, Moher D, Olkin I, et al: Improving the quality of reporting of randomized controlled trials: the CONSORT statement. J Am Med Assoc 1996;272:125-128.

13 Stirman SW, DeRubeis RJ, Crits-Cristoph P, Brody PE: Are samples in randomized controlled trials of psychotherapy representative of community outpatients? A new methodology and initial findings. J Consult Clin Psychol 2003;71:963-972.

14 Mulder RT, Frampton C, Joyce PR, Porter R: Randomised controlled trials in psychiatry. II. Their relationship to clinical practice. Aust NZ J Psychiatry 2003;37:265-269.

15 Clarkin JF, Levy KN, Lenzenweger MF, Kernberg OF: The Personality Disorders Institute/Borderline Personality Disorder Research Foundation randomized control trial for borderline personality disorder: rationale, methods, and patient characteristics. J Pers Dis 2004; 18:52-72.

16 Siever LJ, Torgersen S, Gunderson JG, Livesley WJ, Kendler K: The borderline diagnosis III: identifying endophenotypes for genetic studies. Biol Psychiatry 2002;5:964-968. 